\title{
Performance Improvement of the DSRC System Using a Novel $S$ and $\Pi$-Decision Demapper
}

\author{
Jeich MAR, Chi-Cheng KUO \\ Department of Communications Engineering, Yuan Ze University, Taiwan, China \\ Email: eejmar@saturn.yzu.edu.tw \\ Received March 31, 2009; revised June 20, 2009; accepted June 23, 2009
}

\begin{abstract}
Based on the constellation diagram of the different modulations, a novel $\mathrm{S}$ and $\Pi$-decision rule is designed for the analog demapper of the orthogonal frequency-division multiplexing (OFDM) systems. The dedicated short-range communications (DSRC) system is chosen as an OFDM platform to compare the performance among the proposed $\mathrm{S}$ and $\Pi$-decision decoder, hard-decision and soft-decision decoders. Simulation results demonstrate that both the complexity and performance of S and $\Pi$-decision demapper used for M-ary quadrature amplitude modulation (QAM) OFDM system can be greatly improved. The number of decisions between the received symbol and constellation points can be simplified to look up table $\log _{2} M$ times for M-ary QAM OFDM system.
\end{abstract}

Keywords: S and П-decision Rule, Analog Demapper, DSRC System, OFDM

\section{Introduction}

In the traditional digital communication system where the digital Viterbi decoder is used, the maximum likelihood decision rule is applied to both the demapper and digital hard-decision or soft-decision Viterbi decoder. The soft-decision decoder is the recommended scheme to be used in the digital Viterbi decoder because it provides a coding gain over the hard-decision decoder [1,2]. A simplified algorithm of the soft-decision Viterbi decoder for the 16-quadrature amplitude modulation (QAM) and 64-QAM constellations was presented in [3], which allows the complexity of the demapper to be maintained at almost the same level for all the possible modes of HIPERLAN/2. In [4], it presented that for M-ary QAM systems the complexity of the demapper in a soft-decision Viterbi decoder used for bit-interleaved coded modulation can be significantly lowered without compromising the performance. Four types of analog-input Viterbi decoders are described and compared in [5], where the analog-to-digital converter (A/D) converter is included as part of a digital Viterbi decoder. The analog circuit flaws of the previously used add-compare-select (ACS) chips are included in the comparison. It concludes the analog Viterbi decoder is able to outperform the digital Viterbi decoder, as well as achieve 3-bit or higher decoding resolution. In this paper, we propose a new $\mathrm{S}$ and $\Pi$-decision decoder, where the $\mathrm{S}$ and $\Pi$-decision rules are designed for the analog demapper of the orthogonal frequency-division multiplexing (OFDM) systems. The ACS and path memory (PM) modules, which are parts of the analog Viterbi decoder introduced in [6], are used to perform the Viterbi decoding process. The proposed $\mathrm{S}$ and $\Pi$-decision demapper combined with the digital Viterbi decoder is another alternative to using the $\mathrm{S}$ and $\Pi$-decision decoder. Because analog Viterbi decoder outperforms digital Viterbi decoder and analog very-large-scale integration (VLSI) implementation is in general more area and power efficient than digital implementation, the performance comparison of the $\mathrm{S}$ and $\Pi$-decision decoder using the digital Viterbi decoder is not included in the paper.

The dedicated short-range communications (DSRC) system [7], which employs OFDM technique, provides wireless communications over a short distance between the roadside and high-speed mobile radio units or between high-speed vehicles. The DSRC system will work in a mobile environment with time-varying characteristics. The time-varying fading effect of the DSRC system may not be effectively compensated by using the long symbol training and pilot-based frequency synchronizer [8]. The combined interleaving and convolution coding, which provides the time diversity function, may further improve the performance of the DSRC system. We choose DSRC system as a basis for the performance 
comparison among different decoders. Based on the analog-input Viterbi decoder, the coding gain of the DSRC system achieved by replacing the hard-decision and soft-decision decoders with the proposed $\mathrm{S}$ and $\Pi$-decision decoder will be confirmed with the simulations.

The rest of this paper is organized as follows. In Section II, several channel decoding schemes are described. The proposed $\mathrm{S}$ and $\Pi$-decision decoder is depicted in detail for the binary phase-shift keying (BPSK), quadrature phase-shift keying (QPSK), 16-QAM and 64-QAM OFDM signals of the DSRC systems. Section III briefly describes the base band model of the DSRC system, which is used to compare the bit error rate (BER) performance of the DSRC system for the different decoders. Simulation results are given in Section IV, which show the coding gain achieved by the proposed $\mathrm{S}$ and $\Pi$-decision decoder compared to the hard-decision and soft-decision decoders. Finally, conclusions are drawn in Section V.

\section{S and Decision Decoder for the OFDM Systems}

In the traditional digital communication system, the digital Viterbi decoding uses a maximum likelihood rule which is ideal for an additive white Gaussian noise (AWGN) channel. For a hard-decision Viterbi decoder, the samples matching to a single bit of a code word are quantized to the two levels zero and one, a decision is made as whether each transmitted bit in a code word is zero or one. The coding gain of the soft-decision Viterbi decoder for the hard-decision Viterbi decoder in Rayleigh fading channel increases to about $2 \mathrm{~dB}$ [1]. A four-level discrete symmetric channel model [2] is used for the soft-decision decoder. The demapper assigns one of four values to each received signal. The path metrics in the Viterbi algorithm are calculated by weighting the square of the Hamming distance between the soft-decision and the reference value. The four-level soft-decision Viterbi decoder is almost exactly as shown for the harddecision case with the only difference being the increased number of path metrics.

The block diagram of the proposed $\mathrm{S}$ and $\Pi$-decision decoder for the OFDM system is shown in Figure 1, where it consists of a $\mathrm{S}$ and П-demapper and an analog Viterbi decoder. The $\mathrm{S}$ and $\Pi$-decision decoder is a nonuniform infinite-level quantization decoder. The $\mathrm{S}$ and $\Pi$-decision demapper assigns an analog complex value to the analog Viterbi decoder for each received signal $z(y)$ according to a combination of $\mathrm{S}$ and $\Pi$ functions, as shown in Figure 2. The $\Pi$ function [9], as shown in Figure 2(a), is defined as follows:

$$
\Pi(z(y) ; R, M)=\left\{\begin{array}{cc}
S(z(y) ; M-R, M-R / 2, M) & \text { for } z(y) \leq M \\
1-S(z(y) ; M, M+R / 2, M+R) & \text { for } z(y) \geq M
\end{array}\right.
$$

where $z(y)$ represents either $\hat{I}^{m}(y)$ or $\hat{Q}^{m}(y)$ and $y$ is the received signal after channel compensation. The constellation decoder estimate the $m^{\text {th }}$ symbol gained through the received signal after channel compensation, $\hat{Y}^{m}(y)=\hat{I}^{m}(y)+j \hat{Q}^{m}(y)$, can be found in the signal space diagrams [10] for BPSK, QPSK, 16-QAM and 64-QAM, respectively, where the values of $\hat{I}^{m}(y)$ and $\hat{Q}^{m}(y)$ are serially decoded according to the modulation type. The $\Pi$-function goes to zero at the points

$$
z(y)=M \pm R
$$

while the $\Pi$-function goes to 0.5 at the crossover points

$$
z(y)=M \pm \frac{R}{2}
$$

Notice the parameter $R$ is now equal to one, which is the total width at the crossover points; parameter $M$ is now equal to zero, which is the middle point of the $\Pi$-function. The S-function, as shown in Figure 2(b), is defined as follows:

$$
S(z(y) ; \alpha, \beta, \gamma)=\left\{\begin{array}{cc}
0 & \text { for } z(y) \leq \alpha \\
2\left(\frac{z(y)-\alpha}{\gamma-\alpha}\right)^{2} & \text { for } \alpha \leq z(y) \leq \beta \\
1-2\left(\frac{z(y)-\gamma}{\gamma-\alpha}\right)^{2} & \text { for } \beta \leq z(y) \leq \gamma \\
1 & \text { for } z(y) \geq \gamma
\end{array}\right.
$$

For BPSK modulation, the value of $\hat{I}^{m}(y)$ is in the interval of $(-1,1)$ of constellation diagram. From BPSK constellation diagram, the original one bit binary data $\left(b_{0}\right)$ is decided as $\hat{b}_{0}$ using the S-decision rule. Using (4) for $\alpha=-1, \beta=0$ and $\gamma=1$, the values of $\hat{b}_{0}$ are produced as follows: $\quad \hat{b}_{0}=-1 \quad$ for $z(y) \leqq-1 ; \quad \hat{b}_{0}=1$ for $z(y) \geqq 1$; $\hat{b}_{0}=S(z(y))$ for $-1<z(y)<1$.

For QPSK, the values of $\hat{I}^{m}(y)$ and $\hat{Q}^{m}(y)$ in the constellation diagram are found in the intervals $(-1,1)$. The original two-bit vector $\vec{b}^{(2)}=\left(\mathrm{b}_{0}, \mathrm{~b}_{1}\right)$ is also estimated using the S-function as a decision rule. The $\mathrm{S}$-decision rule in (4) for $\alpha=-1, \beta=0$ and $\gamma=1$ is used to determine $\hat{b}_{0}$ from the received I-channel signal part $\hat{I}^{m}(y)$ and determine $\hat{b}_{1}$ from the Q-channel signal part $\hat{Q}^{m}(y)$. 


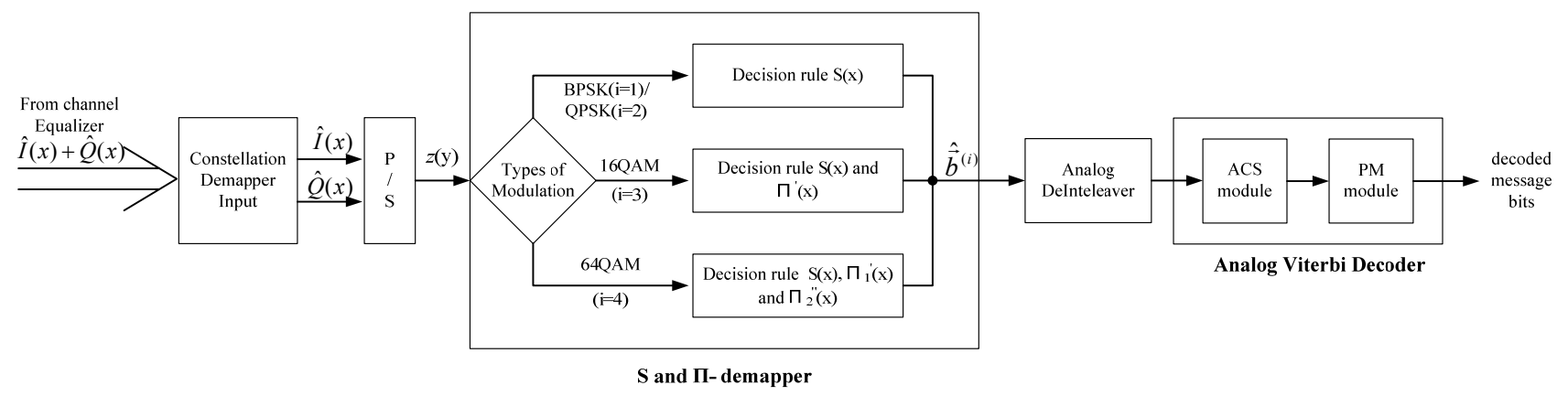

Figure 1. Block diagram of the $S$ and $\Pi$-decision decoder.

The constellation diagram of 16-QAM is shown in Figure 3, where the values of $\hat{I}^{m}(y)$ and $\hat{Q}^{m}(y)$ for 16-QAM modulation are found in the intervals of $(-3,-1$, $1,3)$, respectively. The message points in each quadrant are assigned with Gray-encoded four-bit vector $\vec{b}^{(3)}=$ $\left(b_{0} b_{1} b_{2} b_{3}\right)$. The first two bits $\left(b_{0} b_{1}\right)$ and last two bits $\left(\mathrm{b}_{2} \mathrm{~b}_{3}\right)$ in $\vec{b}^{(3)}$ are transmitted in I and Q-channel, respectively. Both first two bits (boldface) of the I-channel from left to right message points and last two bits (Normal) of the Q-channel from bottom to top message points have the same 8-bit pattern 00011110 in Figure 3. The first four odd bits are 0011 and the second four even bits are 0110 can be estimated by using the $\mathrm{S}$ and $\Pi$-decision rule, respectively, as shown in Figure 4. The $\Pi$-decision rule as shown in Figure 4(a) is defined as

$$
\Pi^{\prime}(z(y))=\left\{\begin{array}{cc}
0 & z(y) \leq-3 \\
S(z(y) ;-3,-2,-1) & -3 \leq z(y) \leq-1 \\
1 & -1 \leq z(y) \leq 1 \\
1-S(z(y) ; 1,2,3) & 1 \leq z(y) \leq 3 \\
0 & z(y) \geq 3
\end{array}\right.
$$

where the S-function is defined in (4). The first two bits $\left(\begin{array}{ll}\hat{b}_{0} & \hat{b}_{1}\end{array}\right)$ and the last two bits $\left(\begin{array}{ll}\hat{b}_{2} & \hat{b}_{3}\end{array}\right)$ of the demapper output for each message point are estimated from the values of $\hat{I}^{m}(y)$ and $\hat{Q}^{m}(y)$, respectively. The S-decision rule is used to determine $\hat{b}_{0}$ and $\hat{b}_{2}$ and the $\Pi$ '-decision rule is used to determine $\hat{b}_{1}$ and $\hat{b}_{3}$. The number of decision needed to obtain $\mathrm{S}$ and $\Pi$-decision demapper output in 16-QAM OFDM system is four.

The same design principle of the $S$ and $\Pi$-decision demapper used for 16-QAM is applied for 64-QAM. The values of $\hat{I}^{m}(y)$ and $\hat{Q}^{m}(y)$ in the constellation diagram for 64-QAM modulation are found in the intervals of $(-7,-5,-3,-1,1,3,5,7)$, respectively. Similarly, the six-bit vector $\hat{\bar{b}}^{(4)}=\left(\begin{array}{llllll}\hat{b}_{0} & \hat{b}_{1} & \hat{b}_{2} & \hat{b}_{3} & \hat{b}_{4} & \hat{b}_{5}\end{array}\right)$ for each message point of 64-QAM modulation is de

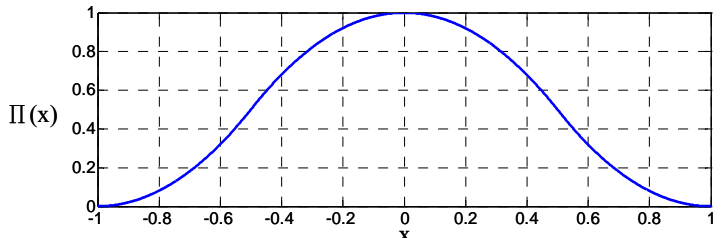

(a)

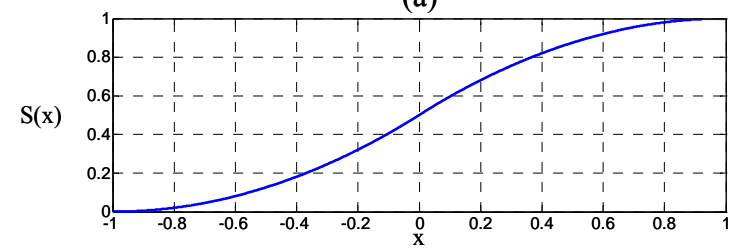

(b)

Figure 2. (a) $\Pi$-decision rule; (b) $S$-decision rule.

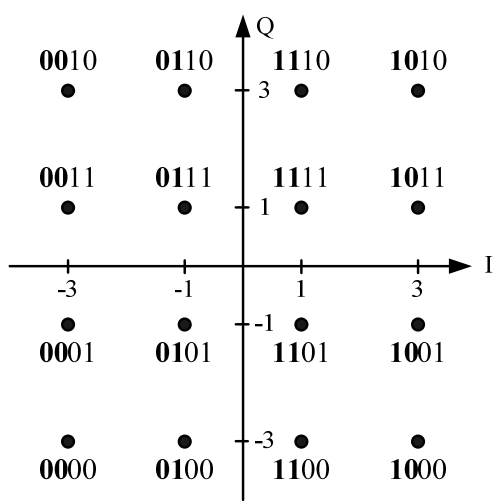

Figure 3. The constellation diagram of 16-QAM.
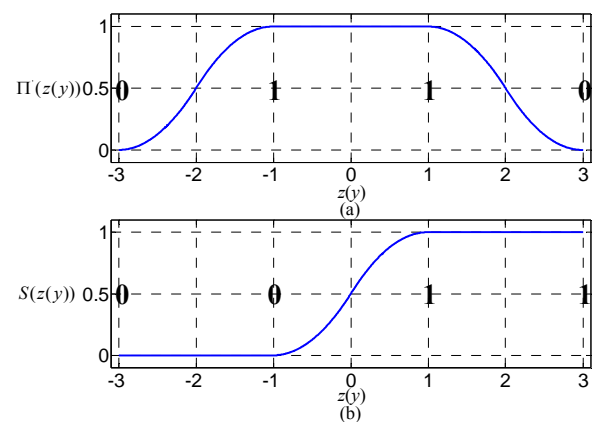

Figure 4. (a) ח'-decision rule; (b) S-decision rule for 16-QAM. 
termined using the $\Pi_{1}^{\prime \prime}, \Pi_{2}^{\prime \prime}$ and S-decision rules, which are designed with 24-bit pattern 000001011 010110111101 100, which is generated from first three bits from left to right message points and last three bits from bottom to top message points, for both I and Q-channels. The first 8-bit pattern $\begin{array}{lllllllll}0 & 0 & 0 & 0 & 1 & 1 & 1 & 1\end{array}$ can be determined from the $\mathrm{S}$-decision rules as shown in Figure 5(a). The second middle 8-bit pattern 00011 1100 can be estimated by using the $\Pi_{1}^{\prime \prime}$-decision function which0 is defined as

$$
\Pi_{1}^{\prime \prime}(z(y))=\left\{\begin{array}{cc}
0 & z(y) \leq-5 \\
S(z(y) ;-5,-4,-3) & -5 \leq z(y) \leq-3 \\
1 & -3 \leq z(y) \leq 3 \\
1-S(z(y) ; 3,4,5) & 3 \leq z(y) \leq 5 \\
0 & z(y) \geq 5
\end{array}\right.
$$

The third 8-bit pattern 0111000110 can be estimated from the $\Pi_{2}^{\prime \prime}$-decision rule as shown in Figure 5(b), which is defined as

$$
\Pi_{2}(z(y))=\left\{\begin{array}{cc}
0 & z(y) \leq-7 \\
S(z(y) ;-7,-6,-5) & -7 \leq z(y) \leq-5 \\
1 & -5 \leq z(y) \leq 3 \\
1-S(z(y) ;-3,-2,-1) & -3 \leq z(y) \leq-1 \\
0 & -1 \leq z(y) \leq 1 \\
S(z(y) ; 1,2,3) & 1 \leq z(y) \leq 3 \\
1 & 3 \leq z(y) \leq 5 \\
1-S(z(y) ; 5,6,7) & 5 \leq z(y) \leq 7 \\
0 & 7 \leq z(y)
\end{array}\right.
$$

The first three bits $\left(\begin{array}{lll}\hat{b}_{0} & \hat{b}_{1} & \hat{b}_{2}\end{array}\right)$ and the last three bits $\left(\begin{array}{lll}\hat{b}_{3} & \hat{b}_{4} & \hat{b}_{5}\end{array}\right)$ of each message point are estimated from the values of $\hat{I}^{m}(y)$ and $\hat{Q}^{m}(y)$, respectively. The S-decision rule is used to determine $\hat{b}_{0}$ and $\hat{b}_{3}$,

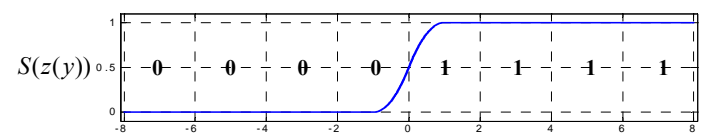

(a)

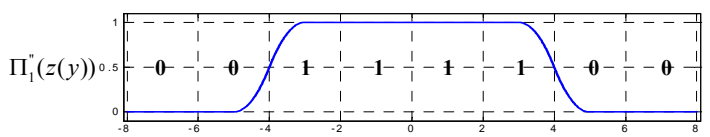

(b)

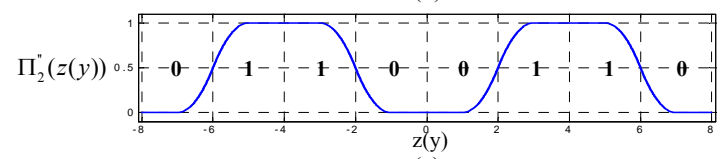

(c)

Figure 5. (a) $S$-decision rule; (b) $\Pi_{1}^{\prime \prime}$-decision rule (c) $\Pi_{2}^{\prime \prime}$ decision rule for 64-QAM. the $\Pi_{1}^{\prime \prime}$-decision rule is used to determine $\hat{b}_{1}$ and $\hat{b}_{4}$ and the $\Pi_{2}^{\prime \prime}$-decision rule is used to determine $\hat{b}_{2}$ and $\hat{b}_{5}$. The number of decisions needed for $\mathrm{S}$ and $\Pi$-demapper in 64-QAM OFDM system is six. It is concluded that for Gray encoded M-ary QAM OFDM systems, the number of decisions for analog $\mathrm{S}$ and $\Pi$-demapper can be reduced to $\log _{2} M$. If the $\mathrm{S}, \Pi, \Pi^{\prime}$ and $\Pi "$ decision rules are calculated and stored in the table, each demapper decision can be simplified as to look up table. The analog Viterbi decoder designed in Figure 1 is a 64-state decoder with a trace back length of 24, 48, 96 and 144 for BPSK, QPSK, 16-QAM and 64-QAM, respectively. The analog complex values of the constellation decoding vectors $\left(\hat{\vec{b}}^{(i)}\right)$ gained through the combinational $\mathrm{S}$ and $\Pi$-decision rules are input to analog deinterleaver and analog Viterbi decoder in turn. The analog deinterleaver [11] permutes the analog demapper output according to the switching order performed in the interleaver. The analog Viterbi decoder consists of the analog ACS module and a digital PM module [6]. ACS module performs the calculation of the analog path metrics. The transmitted message bits are decoded by PM module using the trace back through the trellis architecture. The decoding algorithm and the sequence control for analog Viterbi decoder remain identical with the digital Viterbi decoder.

\section{Base Band Model of the DSRC System}

The block diagram of the DSRC system is shown in Figure 6. The protocol data unit (PDU) trains are applied to the physical layer for transmission. A 127 pseudorandom sequence is used to scramble the data out of the binary sequence before the convolutional encoding. The purpose of the scrambler is to prevent a long sequence of $1 \mathrm{~s}$ or 0 s to aid the timing recovery at the receiver. The generator polynomial [10] of the pseudorandom sequence is

$$
g(D)=D+D^{4}+D^{7}
$$

where $D$ is the unit-delay. The different initialization value is decided by the first 7 bits of each PDU train. The scrambled data sequence is encoded with a rate $1 / 2$ convolutional code with the generator polynomial $\mathrm{g}^{(1)}(D)$ for the upper connection and $\mathrm{g}^{(2)}(D)$ for the lower connection as follows:

$$
\begin{aligned}
& g^{(1)}(D)=1+D^{2}+D^{3}+D^{5}+D^{6} \\
& g^{(2)}(D)=1+D+D^{2}+D^{3}+D^{6}
\end{aligned}
$$

where $D$ is the unit-delay for convolutional codes and the lowest-order term in the polynomial matches the input stage of the shift register. The puncturing pattern [10] is 


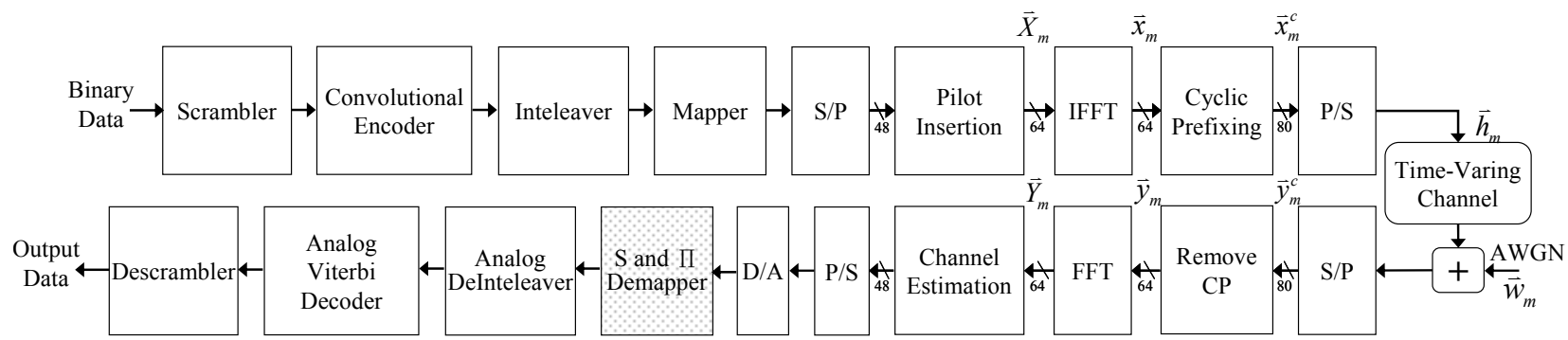

Figure 6. The block diagram of the DSRC base band model.

used to make a rate $3 / 4$ convolutional code from the rate $1 / 2$ convolutional code. A convolutional code may correct many well-spaced errors, while being unable to handle an error burst introduced by the fading channel. The block interleaver or deinterleaver pair [10] applied to the DSRC system can spread the burst error across onto nonadjacent subcarriers and mapped alternately onto less and more significant bits of the constellation. After processing the scrambler, convolution encoder and interleaver, followed by mapping to BPSK or QAM constellation points, the transmitting data stream is divided into several parallel bit streams. An OFDM signal is built using an 64-points inverse fast Fourier transform (IFFT). The input vector to the IFFT is given as

$$
\vec{X}_{m}=\left[X_{m, 0}, X_{m, 1}, \ldots, X_{m, N-1}\right]^{T}
$$

where $X_{m, k}$ represents the $k^{\text {th }}$ subcarrier of the $m^{\text {th }}$ OFDM symbol and $N$ is 64 in the DSRC system. The IFFT output signal vector is

$$
\vec{x}_{m}=\left[x_{m, 0}, x_{m, 1}, \ldots, x_{m, N-1}\right]^{T}
$$

where $x_{m, n}$ is the $n^{\text {th }}$ sample point of the $m^{\text {th }}$ OFDM symbol.

$$
x_{m, n}=\frac{1}{N} \sum_{k=0}^{N-1} X_{m, k} \exp (j 2 \pi n k)=\operatorname{IFFT}\left\{X_{m, k}\right\}
$$

The cyclic prefixes (CP), which are produced with the copies of the last parts of the OFDM symbol, are prepended to the front of each vector $\vec{x}_{m}$. The cyclic prefixing output signal vector is represented as

$$
\begin{aligned}
\vec{x}_{m}^{c} & =\left[x_{m, 0}^{c}, x_{m, 1}^{c}, \ldots, x_{m, N+q-1}^{c}\right]^{T} \\
& =\left[x_{m, N-q}, x_{m, N-q+1}, \ldots, x_{m, N-1}, x_{m, 0}, x_{m, 1}, \ldots, x_{m, N-1}\right]^{T}
\end{aligned}
$$

where $x_{m, n}^{c}$ is the $n^{\text {th }}$ sample point of the $m^{\text {th }}$ OFDM symbol and $q$ is the length of the CP. Therefore, the received signal vector is given by

$$
\vec{y}_{m}^{c}=\vec{x}_{m}^{c} \otimes \vec{h}_{m}+\vec{w}_{m}=\left[y_{m, 0}^{c}, y_{m, 1}^{c}, \ldots, y_{m, N+q-1}^{c}\right]^{T}
$$

Where $\otimes$ stands for linear convolution, $\vec{h}_{m}$ and $\vec{w}_{m}$ are the channel impulse response vector and the additive white Gaussian noise (AWGN) vector for the $m^{\text {th }}$
OFDM symbol, respectively. $y_{m, n}^{c}$ is the $n^{\text {th }}$ sample point of the $m^{\text {th }}$ OFDM symbol in the $m^{\text {th }}$ received signal vector $\vec{y}_{m}^{c}$. The channel impulse response vector $\vec{h}_{m}=\left[h_{m, 0}, h_{m, 1}, \ldots, h_{m, N-1}\right]^{T}$ can be represented by [12]:

$$
h_{m, n}=\sum_{i=0}^{\gamma-1} h_{i}^{m} e^{j \frac{2 \pi}{N} f_{D_{i}} T n} \delta\left(\lambda-\tau_{i}\right), 0 \leq n \leq N-1
$$

where $h_{i}^{m}$ is the complex impulse response of the $m^{\text {th }}$ OFDM symbol in the $i^{\text {th }}$ path; $f_{D i}$ is the $i^{\text {th }}$-path Doppler frequency shift, which may cause intercarrier interference (ICI) for the received signals; $T$ is the sample period; $\lambda$ is the delay spread index; and $\tau_{i}$ is the $i^{\text {th }}$-path delay time normalized by sampling time.

After removing the $\mathrm{CP}$, the received signal vector $\vec{y}_{m}$ is

$$
\vec{y}_{m}=\left[y_{m, 0}, y_{m, 1}, \ldots, y_{m, N-1}\right]^{T}=\left[y_{m, q}^{c}, y_{m, q+1}^{c}, \ldots, y_{m, N+q-1}^{c}\right]^{T}
$$

where $y_{m, n}$ is the $n^{\text {th }}$ sample point of the $m^{\text {th }}$ OFDM symbol. The demodulated received signal vector is

$$
\vec{Y}_{m}=\left[Y_{m, 0}, Y_{m, 1}, \ldots, Y_{m, N-1}\right]^{T}
$$

where

$$
Y_{m, k}=\sum_{n=0}^{N-1} y_{m, n} e^{-j \frac{2 \pi}{N} n k}=\mathrm{FFT}\left\{y_{m, n}\right\}
$$

Suppose the guard interval is longer than the length of the channel impulse response, that is, there is no intersymbol interference between the OFDM symbols, the demodulated sample vector $\vec{Y}_{m}$ can then be represented as [13]

$$
\begin{gathered}
\vec{Y}_{m}=\vec{X}_{m} \vec{H}_{m}+\vec{I}_{m}+\vec{W}_{m} \\
\vec{H}_{m}=\left[H_{m, 0}, H_{m, 1}, \ldots, H_{m, N-1}\right]^{T} \\
\vec{I}_{m}=\left[I_{m, 0}, I_{m, 1}, \ldots, I_{m, N-1}\right]^{T} \\
H_{m, k}=\sum_{i=0}^{\gamma-1} h_{i}^{m} e^{j \pi f_{D_{i} T} T} \frac{\sin \left(\pi f_{D_{i}} T\right)}{\pi f_{D_{i}} T} e^{j \frac{2 \pi \tau_{i}}{N} l}, 0 \leq k \leq N-1
\end{gathered}
$$




$$
I_{m, k}=\frac{1}{N} \sum_{i=0}^{\gamma-1} \sum_{\substack{K=0 \\ K \neq k}}^{N-1} h_{i}^{m} X_{m, k} \frac{1-e^{j 2 \pi\left(f_{D_{i}}-k+K\right)}}{1-e^{j \frac{2 \pi}{N}\left(f_{D_{i}}-k+K\right)}} e^{-j \frac{2 \pi \tau_{i}}{N} K}, 0 \leq k \leq N-1
$$

where $\vec{W}_{m}=F F T\left\{\vec{w}_{m}\right\} . H_{m, k}$ is recognized as the accurate channel frequency response at the $k^{\text {th }}$ subcarrier of the $m^{\text {th }}$ OFDM symbol, which is independent of the transmitted signals $X_{m, k} . I_{m, k}$ is the ICI part of the received signal at the $k^{\text {th }}$ subcarrier of the $m^{\text {th }}$ OFDM symbol, depending on the signal values $X_{m, k}$ modulated on all subcarriers.

On the highway, the maximum vehicle speed is 200 $\mathrm{km} / \mathrm{hr}$. The DSRC system needs more robust frequency and phase synchronization technology. Four uniform pilot subcarriers, which are inserted in the positions of the $6^{\text {th }}$, $20^{\text {th }}, 34^{\text {th }}$, and $48^{\text {th }}$ subcarriers for each of the transmitted DSRC data symbols, are applied for the DSRC receiver to estimate the frequency and track the phase of the received signals. A pilot-based frequency synchronizer mechanism including least squares estimation (LSE) and interpolation is used for equalizing the pilot signal-aided frequency and phase synchronization [14].

\section{Simulations}

Packet detection, timing synchronization and coarse frequency offset estimation of the DSRC receiver are performed according to the algorithms provided in [15]. The simulations focus on comparing the DSRC system performance among the proposed $\mathrm{S}$ and $\Pi$-decision decoder, hard-decision and soft-decision decoders. The DSRC system is specified in the $5.85-5.925 \mathrm{GHz}$ ITS radio services band. In a DSRC system, one frame has 100 OFDM symbols [7]. The total number of subcarriers is 64 including four uniformly distributed pilot subcarriers and 12 guard subcarriers. According to the IEEE $802.11 \mathrm{p}$ standard, the minimum input signal to noise ratio values of the DSRC receiver for BPSK OFDM (3Mbps), QPSK OFDM (6Mbps), 16-QAM OFDM (12Mbps), 16-QAM OFDM (18Mbps), 64-QAM OFDM (24Mbps) and 64-QAM OFDM (27Mbps) modulations are 9, 12, 17, 24, 25 and $27 \mathrm{~dB}$ respectively, which are used as a basis for evaluating the receiver performance. The $3 \mathrm{Mbps}, 6 \mathrm{Mbps}$, and $12 \mathrm{Mbps}$ data transmission rates are made by using $1 / 2$-rate convolutional code. The $18 \mathrm{Mbps}$ and $27 \mathrm{Mbps}$ data transmission rates are produced by using 3/4-rate convolutional code. The $24 \mathrm{Mbps}$ data transmission rate is produced by $2 / 3$-rate convolutional code. The quantization loss for the digital decoding is also considered in the decoding BERs of Figures 9 and 12.

Based on analysis results in [16], the quantization loss for convolutional decoding relative to the continuous case for two-level, four-level and eight-level digital Viterbi decoders are evaluated as 7.73, 1.78, and $0.52 \mathrm{~dB}$ with the $1 / 2$-rate convolutional code; $11.43,2.19$ and $0.55 \mathrm{~dB}$ with the 3/4-rate convolutional code. These losses remain roughly constant across the range of BER plotted. Referring to the SPICE simulation results in [6], the loss of real analog Viterbi decoder with 0.002 ACS noise and $10 \%$ comparator offset is less than $0.2 \mathrm{~dB}$ roughly compared with the ideal analog Viterbi decoders. Therefore, the ACS noise and the comparator offset will not be considered in the following simulations.

In the DSRC system, the coherence time $T_{c}$ is calculated by

$$
T_{c}=\frac{0.423}{f_{D}}=\frac{0.423 \lambda}{v_{D}}=\frac{0.423 c}{v_{D} f_{c}}=645.3 \mu \mathrm{sec}
$$

where $f_{D}=f_{D i}$ for $i=1$ and 2. The maximum Doppler shift is given by $f_{D}=v_{D} / \lambda$, where $v_{D}$ is the maximum vehicle speed, $f_{c}$ is the carrier frequency, and $c$ is the velocity of light. Jakes' channel model [17] is used to produce a time- varying Rayleigh fading channel simulator. The effects of AWGN and carrier frequency shift are also considered in the DSRC channel. Simulations are carried out for the vehicle speed $v_{D}=200 \mathrm{~km} / \mathrm{hr}$, delay spread $\tau$ $=200 \mathrm{nsec}, 100$ data symbols and different decision Viterbi decoder.

Figure 7 shows when the delay spread exceeds 150 $n$ sec, the severer frequency selective channel fading will be caused by reducing coherent bandwidth. The BER performance of the DSRC receiver with BPSK OFDM (3Mbps), QPSK OFDM (6Mbps), 16-QAM OFDM (12Mbps), 16-QAM OFDM (18Mbps), 64-QAM OFDM (24Mbps) and 64-QAM OFDM (27Mbps) modulations are shown in Figure 8-12 respectively. Figure 8 shows the BER of QPSK OFDM modulations for Viterbi decoders using three different decision rules are reduced to

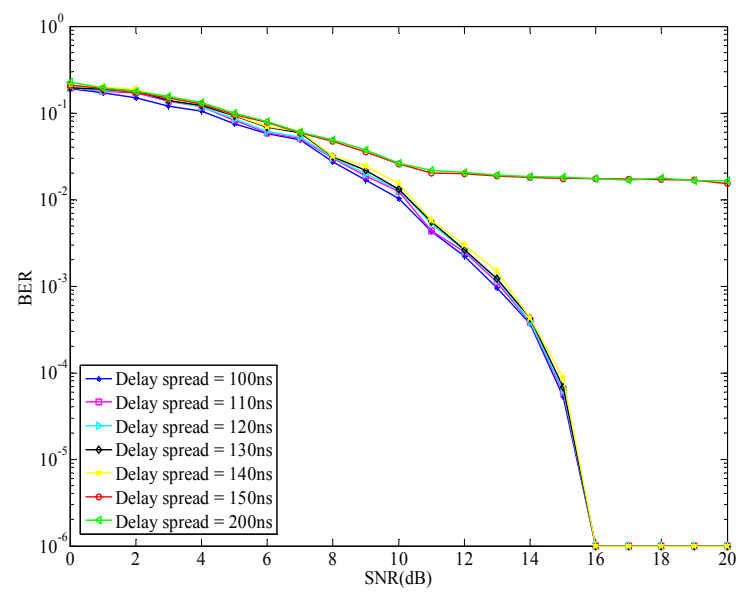

Figure 7. BERs of the DSRC system using pilot subcarrieraided equalizer in different delay spread for 16-QAM OFDM modulations. 


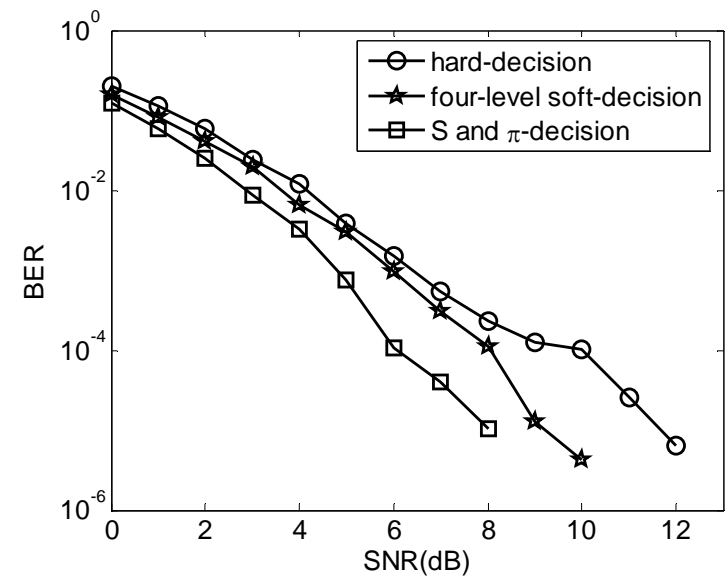

Figure 8. Comparisons of BERs of the QPSK DSRC system (6 Mbits/sec; $200 \mathrm{~km} / \mathrm{h}$; code-rate is $1 / 2$ ) in terms of the different decision decoders.

less than $10^{-5}$ at the minimum signal-to-noise ratio (SNR), which meets the requirements specified in the IEEE $802.11 \mathrm{p}$ standard.

When the quantization loss for the convolutional decoding is considered, the BER of 16-QAM OFDM DSRC system is shown in Figure 9, where using a hard- decision Viterbi decoder is higher than $10^{-5}$ at the minimum SNR (17dB) for the case of the 12Mbps data transmission rate. The 16-QAM OFDM DSRC system using the four-level and eight-level soft-decision and analog Viterbi decoders will be reduced to less than $10^{-5}$ at the minimum SNR $(17 \mathrm{~dB})$, which meet the requirements specified in the IEEE802.11p standard. It is noted the $S$ and $\Pi$-decision decoders results in a coding gain of $1.5 \mathrm{~dB}$ and $5.2 \mathrm{~dB}$ compared to the eight-level and four-level soft-decision decoders, respectively, when the quantization loss for the convolutional decoding is considered. Figure 10 shows the BER of 16-QAM DSRC system using the hard-decision decoder cannot be lower than $10^{-5}$, when the data transmission rate increases to $18 \mathrm{Mbps}$. The hard-decision curve has a floor that is generated by the hard-decision loss under the two-ray Rayleigh fading channel environment. The 16-QAM DSRC system using both the soft-decision decoder and the $\mathrm{S}$ and $\Pi$-decision decoders will reduce the BER to less than $10^{-5}$ at the minimum SNR $(24 \mathrm{~dB})$, which meets the requirements specified in the IEEE $802.11 \mathrm{p}$ standard. The $\mathrm{S}$ and $\Pi$-decision decoder has a $1.5 \mathrm{~dB}$ coding gain compared with the four-level soft-decision decoder.

Figure 11 shows the BER of 64-QAM DSRC system under the conditions of $18 \mathrm{Mbits} / \mathrm{sec}$ data rate and $200 \mathrm{~km} / \mathrm{h}$ vehicle speed cannot be lower than $10^{-5}$ for three different Viterbi decoders. All three BER curves have the floors that are caused by the high-order 64QAM modulation DSRC system operated under the fast fading channel. The BER of 64-QAM DSRC system is shown in Figure 12, where the quantization loss for the

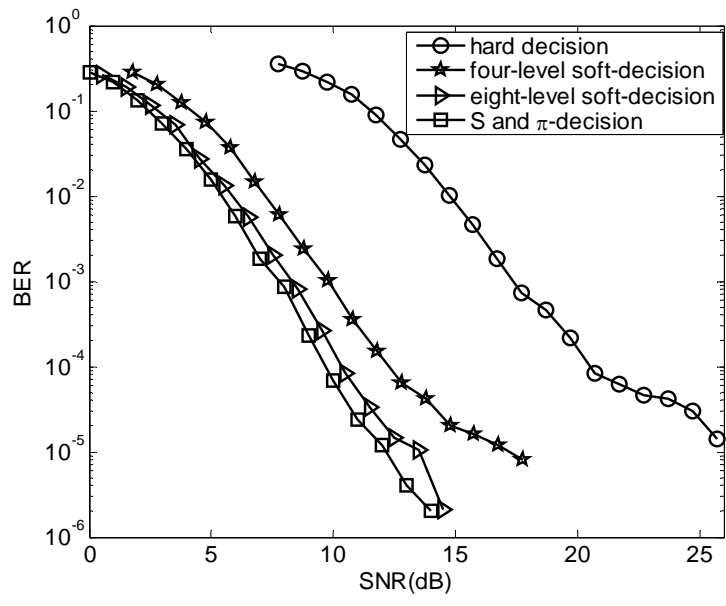

Figure 9. Comparisons of BERs of the 16-QAM DSRC system $(12 \mathrm{Mbits} / \mathrm{sec} ; 200 \mathrm{~km} / \mathrm{h}$; code-rate is $1 / 2)$ in terms of the different decision decoders with quantization loss.

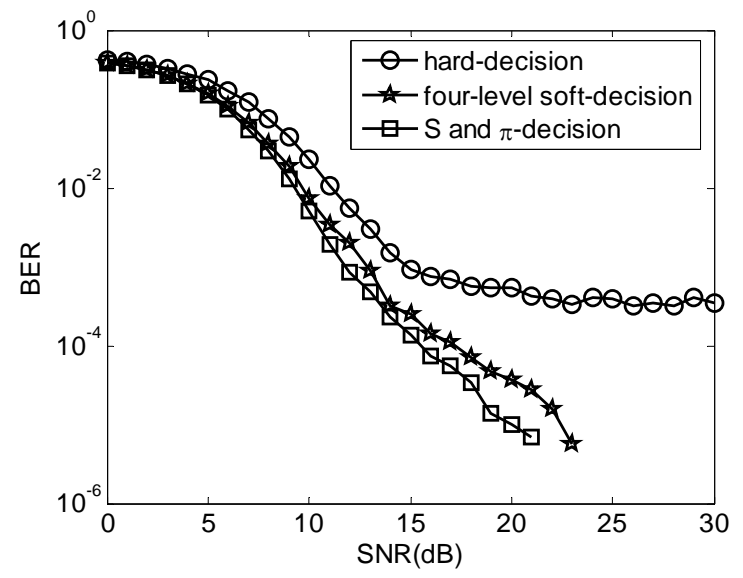

Figure 10. Comparisons of BERs of the 16-QAM DSRC system (18 Mbits $/ \mathrm{sec} ; 200 \mathrm{~km} / \mathrm{h}$; code-rate is $3 / 4)$ in terms of the different decision decoders.

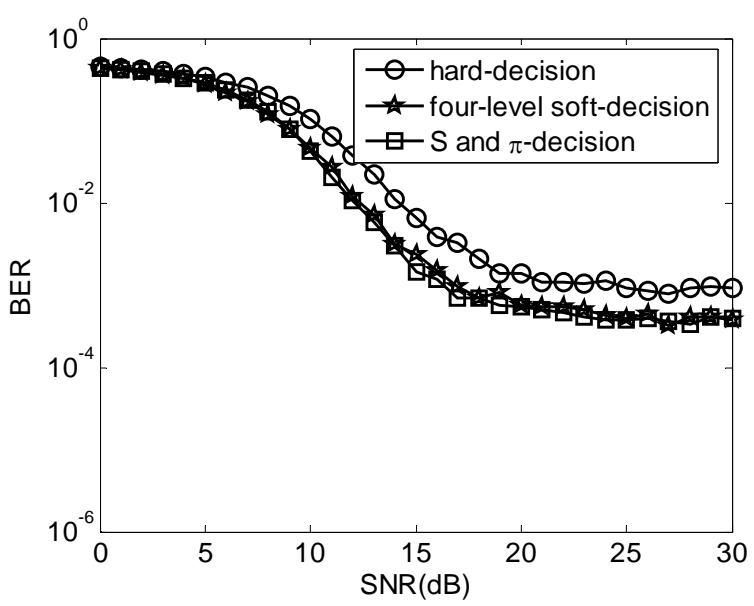

Figure 11. Comparisons of BERs of the 64-QAM DSRC system (24 Mbits/sec; $200 \mathrm{~km} / \mathrm{h}$; code-rate is $2 / 3$ ) in terms of the different decision decoders. 


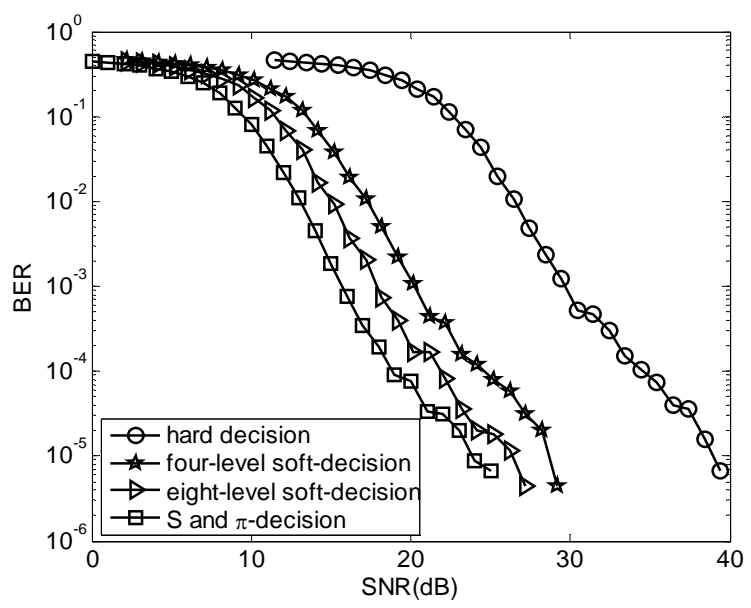

Figure 12. Comparisons of BERs of the 64-QAM DSRC system (27 Mbits $/ \mathrm{sec} ; 120 \mathrm{~km} / \mathrm{h}$; code-rate is $3 / 4)$ in terms of the different decision decoders with quantization loss.

convolutional decoding is considered and the vehicle speed reduces to $v_{m}=120 \mathrm{~km} / \mathrm{hr}$. It showed the 64-QAM DSRC system using three different decoders can be reduced to less than $10^{-5}$ at the minimum SNR $(27 \mathrm{~dB})$, which meets the requirements specified in IEEE802.11p standard. The $\mathrm{S}$ and $\Pi$-decision decoder results in a coding gain of $2.5 \mathrm{~dB}$, $5 \mathrm{~dB}$ and $15 \mathrm{~dB}$ compared to the eight-level soft-decision, four-level soft-decision and hard-decision Viterbi decoders, respectively, when the quantization loss for the digital Viterbi decoding is considered.

\section{Conclusions}

A new $\mathrm{S}$ and $\Pi$-decision rule is proposed for the analog demapper of the OFDM system. The DSRC system is chosen as an OFDM platform to compare the performance of the $\mathrm{S}$ and $\Pi$-decision decoder, which consists of an $\mathrm{S}$ and $\Pi$-decision demapper, analog deinterleaver and an analog Viterbi decoder, with hard-decision and softdecision decoders. Simulation results show the coding gain of the $\mathrm{S}$ and $\Pi$-decision decoder relative to four-level and eight-level soft-decision decoders are evaluated as $5.4 \mathrm{~dB}$ and $1.5 \mathrm{~dB}$, respectively, with the 16-QAM DSRC system; and $5 \mathrm{~dB}$ and $2.5 \mathrm{~dB}$ with the 64-QAM DSRC system when the quantization loss for the convolution decoding is considered. Each analog demapper output can be determined by looking-up $\mathrm{S}$ and $\Pi$ table $\log _{2} M$ times for M-ary QAM OFDM systems. Many other applications related to OFDM with the proposed $\mathrm{S}$ and $\Pi$-decision decoder are possible.

\section{Acknowledgement}

The authors would like to acknowledge gratefully the research grants from National Science Council, Taiwan,
NSC 96-2219-E-155-005.

\section{References}

[1] K. M. Lee, D. S. Han, and K. B. Kim, "Performance of the Viterbi decoder for DVB-T in Rayleigh fading channels," IEEE Trans. Consumer Electron., Vol. 44, pp. 994-1000, August 1998.

[2] S. B. Wicker, "Error control systems for digital communication and storage," New Jersey, Prentice-Hall, Inc., 1995.

[3] F. Tosato and P. Bisaglia, "Simplified soft-output demapper for binary interleaved COFDM with application to HIPERLAN/2," in Proc. IEEE Int. Conf. Communications, Vol. 2, pp. 664-668, April 2002.

[4] E. Akay and E. Ayanoglu, "Low complexity decoding of bit-interleaved coded Modulation for M-ary QAM," in Proc. IEEE Int. Conf. Communications, Vol. 2, pp. 901905, June 2004.

[5] K. He and G. Cauwenberghs, "Performance of analog Viterbi decoding," 42nd Midwest Symposium on Circuits and Systems, Vol. 1, August 1999.

[6] K. He and G. Cauwenberghs, "Itergrated 64-state parallel analog Viterbi decoder," ISCAS 2000, Vol. 4, May 2000.

[7] Standard Specification for Telecommunications and Information Exchange Between Roadside and Vehicle System-5Ghz Band Dedicated Short Range Communications (DSRC) Medium Access Control (Mac) and Physical Layer (PHY) Specifications, IEEE802.11p, December 2005.

[8] S. Coleri, M. Ergen, and A. Bahai, "Channel estimation techniques based on pilot arrangement in OFDM systems," IEEE Trans. Broadcast., Vol. 48, pp. 223-229, September 2002.

[9] J. Giarratano and G. Riley, Expert Systems, 2nd. Edition, PWS Publishing Company, 1994.

[10] J. Heiskala and J. Terry, OFDM Wireless LANs: A Theoretical and Practical Guide, Sams, 2001.

[11] V. C. Gaudet, R. J. Gaudet, and P. G Gulak, "Programmable interleaver design for analog iterative decoders," IEEE Trans. Circuits Syst. II, Vol. 49, pp. 457-464, July 2002.

[12] D. G. Manolakis, V. K. Ingle, and S. M. Kogon, "Statistical and adaptive signal processing," McGraw-Hill, Education, 2000.

[13] Y. Zhao and A. Huang, "A novel channel estimation method for OFDM mobile communication systems based on pilot signals and transform-domain processing," in Proc. IEEE VTC, Vol. 3, pp. 2089-2093, May 1997.

[14] J. Rinnie and M. Renfors, "Pilot spacing in orthogonal frequency division multiplexing systems on practical channels," IEEE Trans. Consumer Electron., Vol. 42, No. 4, pp. 959-962, November 1996.

[15] IEEE 802.11a, IEEE standard for Wireless LAN Medium Access Control (MAC) and Physical Layer (PHY) specifications, 1999.

[16] M. R. G. Butler and A. R. Nix, "Quantization loss for convolutional decoding in Rayleigh-fading channels," IEEE Commun. Lett., Vol. 7, No. 9, pp. 446-448, September 2003.

[17] W. C. Jakes, Microwave Mobile Communications, New York: IEEE Press, 1974. 